\title{
Designing the Shape of Corona Virus Using the PDE Method
}

\author{
Jiyar Jafar Ahmed \\ Department of Mathematics, College of Science, University of Duhok, Duhok-IRAQ.
}

\begin{abstract}
The aim of this study is designing the shape of corona virus (COVID-19) using the partial differential equation (PDE). The technique improvement was based on using an elliptic PDE as well as a set of four boundary conditions. The PDE method can generate surfaces of geometries from a small number of parameters. Also, the shape of the surfaces, which is generated by the PDE method, is based on a boundary representation and it can easily be changed since it is described by data distributed around the boundaries. In this study, the shape of the generated PDE-based representation of a corona virus has been sketched by using MATLAB program. The results showed that the PDE method is appropriate for representing the shape of a corona virus. Besides that, the data, concerning the radius and height from the corona virus, are then used to get four equations. These equations can be used for future prediction in modeling COVID-19. In conclusion, the PDE method can produce smooth parametric surface representations of any given shapes of viruses. The study involves that the PDE method has ability to generate surfaces of complex geometries.
\end{abstract}

Keywords: Partial differential equation, parametric surface, geometric designing, periodic boundary conditions. 2010 MSC: MSC 49K20, 65Mxx.

\section{Introduction}

A group of anonymous cases of pneumonia in Wuhan, China, caused concern of health officials in late December 2019. On 31st December, an alert was issued by the Wuhan Municipal Health Committee, and a rapid response team was sent to Wuhan by the Chinese Center for Disease Control and Prevention (China CDC), and the World Health Organization (WHO) was notified [6, 12, 13, 14]. Possible causes including influenza, avian influenza, adenovirus, acute coronavirus and severe acute respiratory syndrome (SARS-CoV), and coronary syndrome of the Middle East Respiratory Syndrome (MERS-CoV) have been excluded. An epidemiological investigation was involved in the Huanan seafood wholesale market in Wuhan, which has been closed and cleared, and the process of discovering and actively following the active situation has begun $[6,13,15]$. On 7th January, 2020, the pathogen was identified as a new coronavirus, followed by genomic characterization and development of the test method $[3,6,13,14,15]$. The virus is now known as 2019-nCoV, and differs from SARS-CoV and MERS-CoV, but it is closely related $[3,9,15]$. Early cases indicated that COVID-19 (i.e., the new name of the disease caused by a new coronavirus) may be less severe than SARS and MERS. However, the onset of the disease between the rapidly increasing numbers of people and the growing evidence of human-to-human transmission indicates that 2019-nCoV is more infectious than both SARS-CoV and MERSCoV [4, 5, 7, 8, 14]. On 20th January, the

Email address: jiyar.ahmed@uod.ac (Jiyar Jafar Ahmed)

doi:https:/ / doi.org/10.31559/glm2020.8.2.5

Received 10 April 2020 : Accepted 14 June 2020 
China's "National Infectious Diseases Law" of 2019 to change coronary lung disease of the year (COVID19) category B notifies capable disease and the Border Health and Quarantine Law was amended to support the response to the COVID-19 outbreak effort. Then, on 23rd January, the Chinese government started limiting the movement of people to and from Wuhan, and two days later, it announced its commitment to the highest level and mobilized all sectors to respond to the epidemic and prevent further spread of COVID-19. Characterization of the epidemiological features of COVID-19 is crucial for the development and implementation of effective control strategies. Studying the mathematics of corona virus could be one of the most interesting topic because it is directly related to our life and health. A method which is called PDE method is a technique based on using partial differential equation (PDE) [10] which can be used to design corona virus. This method has been introduced in Computer-aided Design (CAD) as a solution of a particular type of elliptic PDEs to generate smooth parametric surfaces based on a boundary representation. This is the easiest and simplest method because it depends on boundary condition (BC) to generate the corona virus in a simple way [10]. The paper has been ordered as section two describes the mathematical theory related with the PDE method and explains the methodology used to generate a parametric corona virus. Section three inlcudes a shape of corona virus as well as its properties. Finally, section four concludes what has been done throughout this work.

\section{THE PDE METHOD}

The PDE method was originally introduced into the area of computer graphics in 1989 and it has been widely used as a surface-modeling technique in many areas especially in graphics and designing, design analysis and optimization [1]. This method produces a parametric surface, $\underline{\chi}(u, v)$ that is generated by solving the fourth order elliptic PDE,

$$
\left(\frac{\partial^{2}}{\partial u^{2}}+\alpha^{2} \frac{\partial^{2}}{\partial v^{2}}\right)^{2 r} \underline{\chi}(u, v)=0
$$

where $\alpha$ is an intrinsic parameter controlling the relative smoothness of the surface in the $u$ direction and $r$ defines the order of the PDE, and they are restricted to $\alpha \geqslant 1$ and $r \geqslant 1$ [2]. equation 2.1 is known as the Biharmonic equation. The analytic solution to equation 2.1 is found using separation of variables subject to four periodic boundary conditions,

$$
\begin{aligned}
& \underline{\chi}(0, v)=\underline{P_{0}}(v), \\
& \underline{\chi}(1, v)=\underline{P_{1}}(v), \\
& \frac{\partial \underline{\chi}}{\partial u}(0, v)=\underline{d_{0}}(v), \\
& \frac{\partial \underline{\chi}}{\partial u}(1, v)=\underline{d_{1}}(v),
\end{aligned}
$$

where $\underline{P_{0}}(v)$ and $\underline{P_{1}}(v)$ define the edge of the surface patch at $u=0$ and $u=1$, respectively, while $\underline{d_{0}}(v)$ and $\underline{d_{1}} \overline{(v)}$ are the values of the normal derivatives at the respective boundary of the surface.

The derivative conditions are defined by the derivative vector along the boundary curves and they determine the overall shape of the surface [11]. The magnitude and the direction of the derivative vector are determined by the difference between each point on the derivative curve and an associated point on the boundary curve.

The solution to equation 2.1 has been found using the separation of variables method. However, the present work is employed the approximated solution of equation 2.1 which has been truncated to $\mathrm{N}$ Fourier modes,

$$
\left.\underline{x}(u, v)=\underline{A_{0}}+\sum_{n=1}^{N} \underline{A_{n}}(u) \cos (n v)+\underline{B}(u) \sin (n v)\right]+\underline{R}(u, v),
$$


where

$$
\begin{gathered}
\underline{A_{0}}=\underline{a_{00}}+\underline{a_{01}} u+\underline{a_{02}} u^{2}+\underline{a_{03}} u^{3}, \\
\underline{A_{n}}=\left(\underline{a_{n 1}}+\underline{a_{n 3}} u\right) e^{n u}+\left(\underline{a_{n 2}}+\underline{a_{n}} u\right) e^{-n u}, \\
\underline{B_{n}}=\left(\underline{b_{n 1}}+\underline{b_{n 3}} u\right) e^{n u}+\left(\underline{b_{n 2}}+\underline{b_{n 4}} u\right) e^{-n u}, \\
\underline{R}=\left(\underline{r_{1}}(v)+\underline{r_{3}}(v) u\right) e^{\beta u}+\left(\underline{r_{2}}(v)+\underline{r_{4}}(v) u\right) e^{-\beta u,},
\end{gathered}
$$

The term $\sum_{n=1}^{\infty}\left[A_{n}(u) \cos (n v)+\underline{B_{n}}(u) \sin (n v)\right]$ defines the position of a point on the surface relative to the spine and $\overline{A_{0}}(u)$ where $a_{00}, a_{01}, \ldots, a_{11}, a_{12}, \ldots, b_{11}, \ldots, b_{N 4}$ are vector-valued constants, is a cubic polynomial of the parameter $u$ and lies inside the periodic surface patch so the solution system defined in equation 2.6. The remainder function $\underline{R}$ is responsible for fully satisfying the original boundary conditions. Thus, the vectors $\underline{r}_{1}, \ldots, \underline{r_{4}}$ and $\beta$ are obtained from the difference between the original boundary conditions and the one satisfied by the first two terms in equation 2.6.

Figure 1(b) illustrates the example of one patch PDE surface describing the outer shape of an apple, while figure 1(a) shows the generating curves of such a surface. The surface is generated when using equation 2.6 with $\mathrm{N}=5$. For the adjacent patch, one surface or part of it needs to be blended to another surface as shown in figure 2.

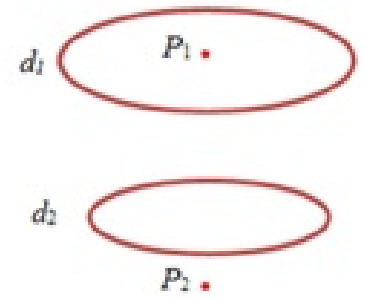

(a)

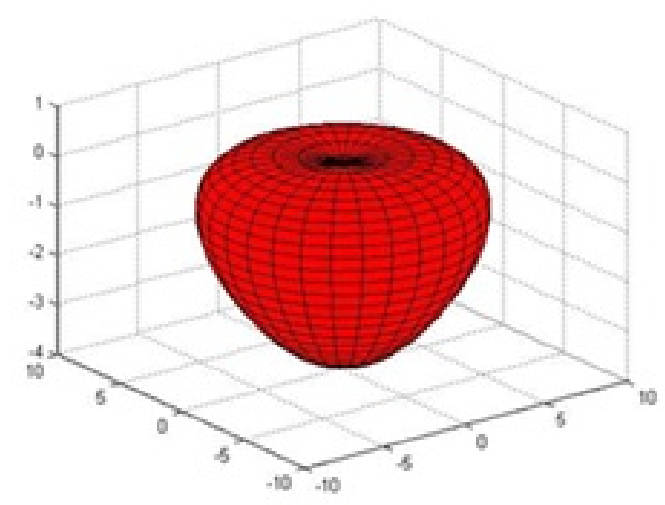

(b)

Figure 1: A parametric apple. (a)The boundary conditions in 3-space and (b) The generated surface shape.

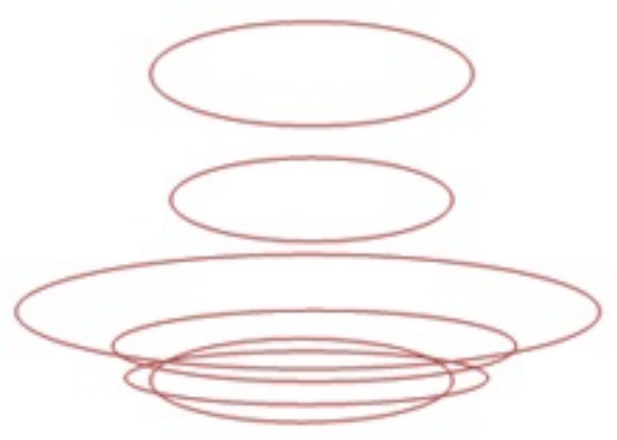

(a)

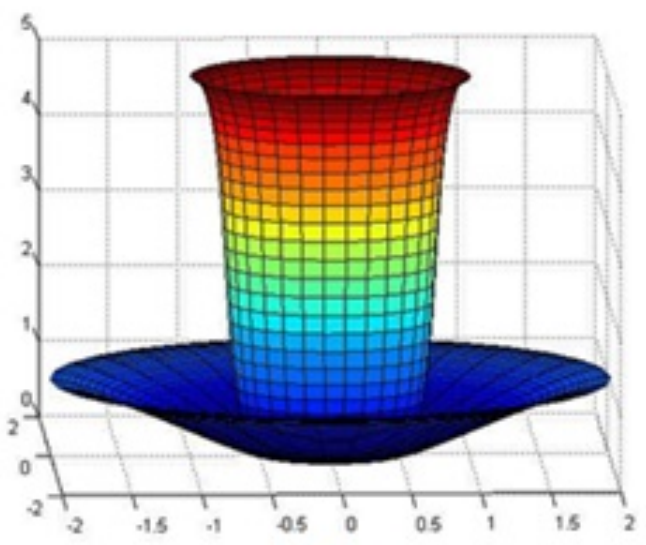

(b)

Figure 2: A PDE-based east tea cup. (a)The boundary curves. (b) The resulting surface shape. 


\subsection{Designing Corona virus}

This section discusses how to use the PDE method for modelling corona virus. The shape of corona virus is a combination between two shapes. One of them is the body and it looks like a circle while the other one is called spikes ending in little blobs coming out of the surface. The geometric model representing the body part of corona virus's shape which is used during this work that has been obtained using seven generating curves to produce a surface collected of two patches. Since every surface patch needs four boundary curves and one curve will be common curve between two patches. On the other hand, for designing the spike part of corona virus which is only one patch that means four boundary curves will be used for sketching this part. The curves are generated using MATLAB. Every curve is represented by several points from 0 to $2 \pi$ and the last point confirms that each curve is closed properly. Every curve represents a circle of a given radius at a particular height.

In order to use the PDE method to design the body part of corona virus, the designing procedures are divided into several steps. First, the top part (Patch one) is generated by designing the bottom part of circle (Patch two) and by using the same way the bottom part is generated by designing the top circle. Combining the top and the bottom circle to get the shape of the body of corona virus. Since the shape of corona virus has many spikes and all spikes have the different center as shown in figure 3.

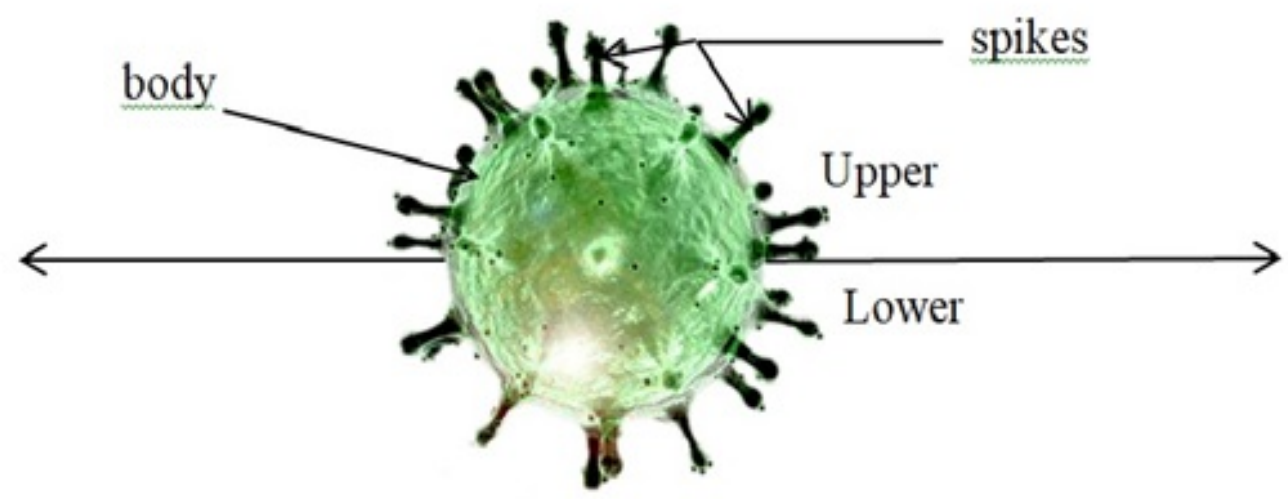

Figure 3: Corona virus.

Only the upper circle will be considered for the geometric representation of the axisymmetric body part of corona virus because this object is symmetric about z-axis. Also the shape of spike part will be designed. Then combining these two parts to get the shape of corona virus. The upper circle and spike part are determined by

$$
r_{i}=a \cos (\phi) \text { and } h_{i}=b \sin (\phi)
$$

where $r_{i}$ is the size, $h_{i}$ is the height, $\phi=[0, \pi]$ and $a$ and $b$ are the radius of circle on $x$-axis and $y$-axis, respectively. Generally, the coordinate of each point on the boundary curves can be written as

$$
\left(x_{0}+r_{i} \cos (v), y_{0}+r_{i} \sin (v), z_{0}+h_{i}\right),
$$

where $0 \leqslant v \leqslant 2 \pi$ and $x_{0}, y_{0}, z_{0}$ represent the center of circle.

Now consider the boundary curve which is used to generate the first patch (Patch one) of the upper circle with $a=30 \mathrm{~mm}$ and $b=30 \mathrm{~mm}$. In particular, the coordinate for boundary curves are

$$
\begin{gathered}
P_{11}=\left(x_{0}+30 \cos (v), y_{0}+30 \sin (v), z_{0}\right), \\
P_{12}=\left(x_{0}, y_{0}, z_{0}+30\right), \\
d_{11}=\left(x_{0}+0.2 \cos (v), y_{0}+0.2 \sin (v), z_{0}+1.4\right),
\end{gathered}
$$




$$
\mathrm{d}_{12}=\left(\mathrm{x}_{0}+(-100) \cos (v), \mathrm{y}_{0}+(-100) \sin (v), z_{0}+(-2)\right),
$$

where $P_{11}, P_{12}, d_{11}$ and $d_{12}$ represent $B C 1, B C 2, B C 3$ and $B C 4$ for patch one respectively. Notice that $B C 1$ is the base, $\mathrm{BC} 2$ is the top, while $\mathrm{BC} 3$ and $\mathrm{BC} 4$ are the body for every shape which can be seen in figure 4.

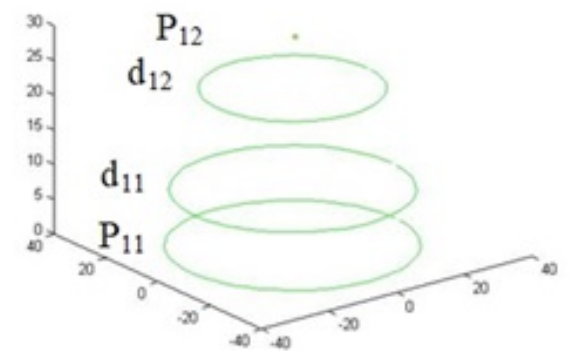

(a)

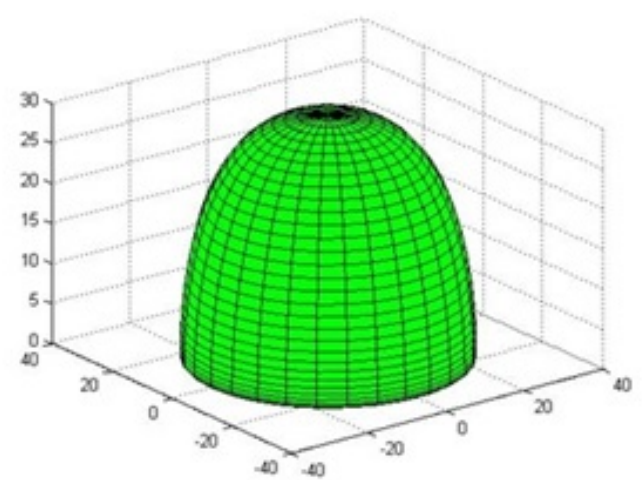

(b)

Figure 4: a) Generating curves in three-dimensional space for upper circle. b) The resulting surface shape of corona virus upper circle.

As displayed in figure $4(a)$, the curve $P_{11}$ is chosen to be the sharing boundary curve where both surface patches meet. The curves in equations (13), (14), (15), and (16) can be reflected to obtain the boundary curves corresponding to the patch two which is lower circle see figure 5.

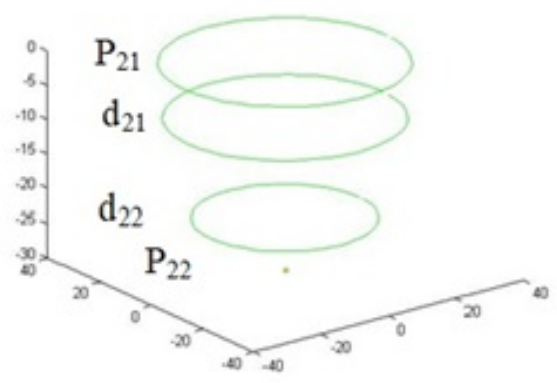

(a)

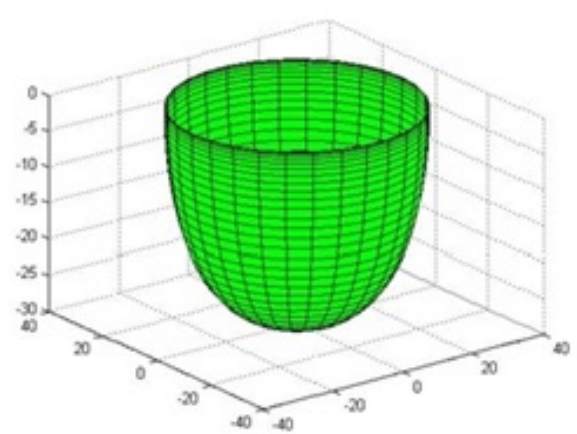

(b)

Figure 5: a) Generating curves in three-dimensional space for lower circle. b) The resulting surface shape of corona virus lower circle.

For designing the body part of corona virus figure 4 and figure 5 are connected. Since each part has four boundary curves but one of them which is $\mathrm{P}_{11}$ or $\mathrm{P}_{21}$ is a common curve between two patches. Therefore, the body part of corona virus has seven boundary curves as shown in figure 6. 


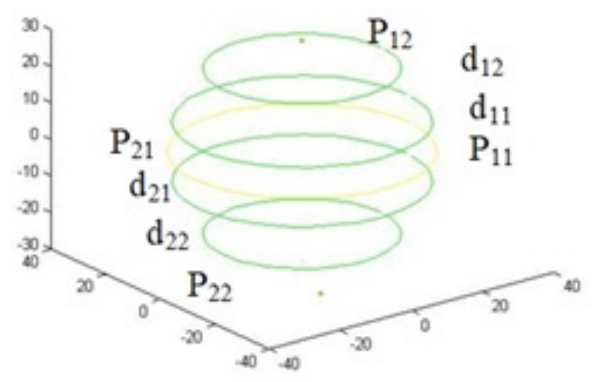

(a)

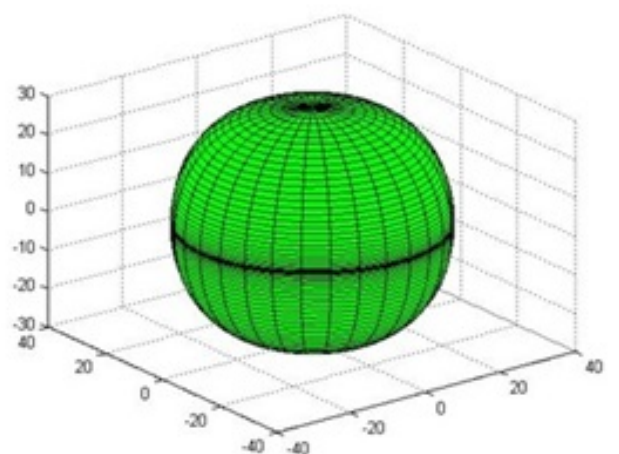

(b)

Figure 6: a) combination between boundary curve one and two. b) shape of the body of corona virus.

The shape of spikes is going to be designed. As it is mentioned before, corona virus has many spikes and all spikes have the different center. Therefore, only a spike needs to be designed. The shape of spike is a part say patch three with four boundary conditions as follows:

$$
\begin{gathered}
P_{31}=\left(x_{0}+2 \cos (v), y_{0}+2 \sin (v), z_{0}+30\right), \\
P_{12}=\left(x_{0}, y_{0}, z_{0}+50\right), \\
d_{31}=\left(x_{0}+0.001 \cos (v), y_{0}+0.001 \sin (v), z_{0}+50\right), \\
d_{32}=\left(x_{0}+(-25) \cos (v), y_{0}+(-25) \sin (v), z_{0}+1\right),
\end{gathered}
$$

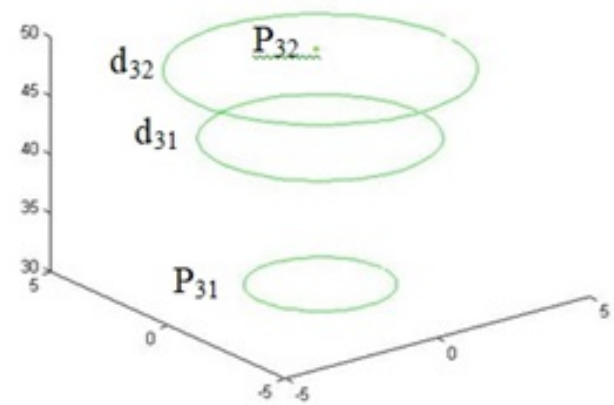

(a)

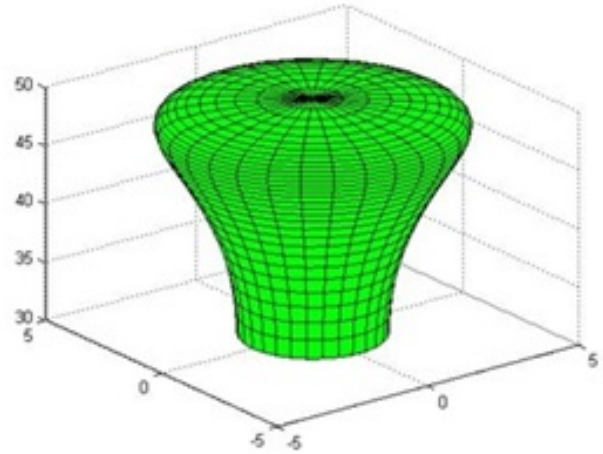

(b)

Figure 7: a) curves for spike. b) The surface shape spike.

In order to obtain the shape of corona virus, the shape of body part, see figure 5, and spike's shape which is figure 6 , will be combined, see figure 7 . 


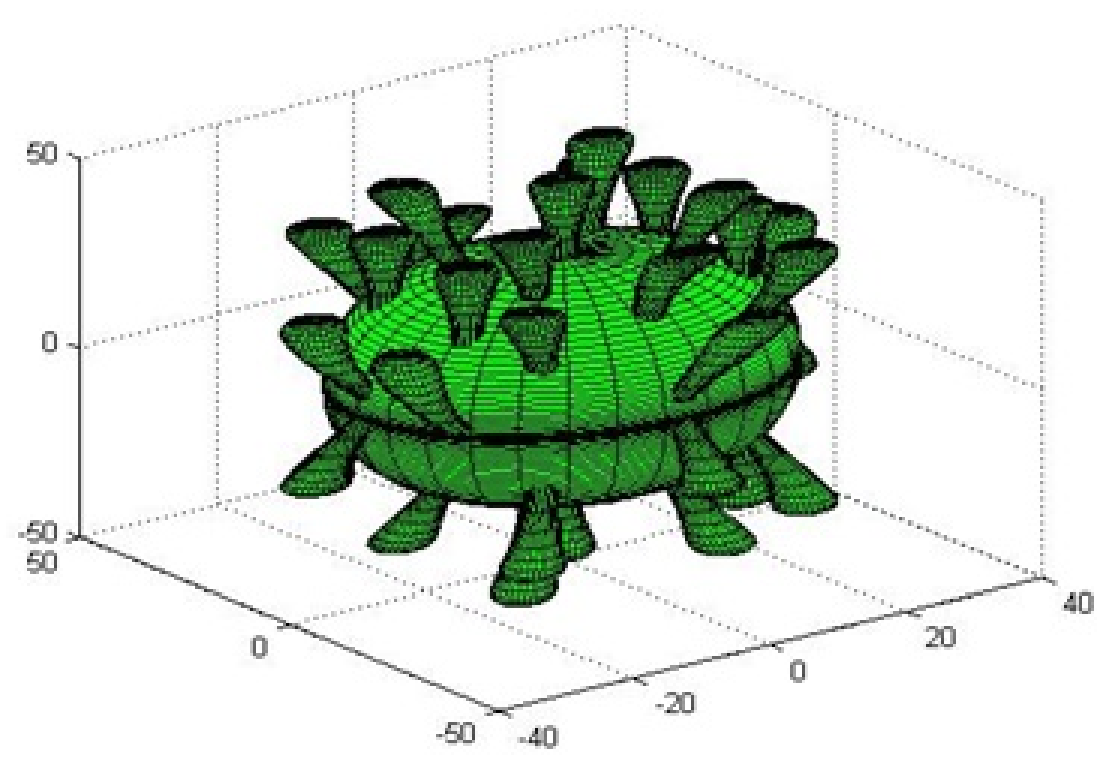

Figure 8: 3D shape of corona virus.

\section{CONCLUSIONS}

This paper has focused on the application of the analytic solution of a particular Elliptic PDE for modeling corona virus. These expressions were used to modify the shape of corona virus to reach the required designs. PDE Method is the best and easiest method as it depends on the boundary condition and it is possible for every geometry to be generated and controlled by few design parameters.

\section{References}

[1] N., Ahmat, G. González Castro, and H. Ugail, Automatic shape optimisation of pharmaceutical tablets using Partial Differential Equations. Computers \& Structures, (2014), 130, 1 - 9. https:/ / doi.org/10.1016/j.compstruc.2013.09.001 2

[2] N., Ahmat, H. Ugail, and G. González Castro, Method of modelling the compaction behaviour of cylindrical pharmaceutical tablets. International Journal of Pharmaceutics, 405(1-2), (2011), 113-121. https:/ / doi.org/10.1016/j.ijpharm.2010.12.006 2

[3] JFW. Chan, KH. Kok, Z. Zhu, H. Chu, KKW. To, SF. Yuan, et al. Genomic characterization of the 2019 novel humanpathogenic coronavirus isolated from a patient with atypical pneumonia after visiting Wuhan. Emerg Microbs Infect. http:/ /dx.doi.org/10.1080/ 22221751.2020.1719902. [2020-01-24]. https:/ / doi.org/10.1080/22221751.2020.1719902 1

[4] JFW. Chan, S. Yuan, KH. Kok, KKW. To, H. Chu, J. Yang, et al. A familial cluster of pneumonia associated with the 2019 novel coronavirus indicating person-to-person transmission: a study of a family cluster. Lancet. http:/ /dx.doi.org/10.1016/S0140-6736(20) 30154-9. [2020-01-24]. 1

[5] C. Huang, Y. Wang, X. Li, L. Ren, J. Zhao, Y. Hu, et al. Clinical features of patients infected with 2019 novel coronavirus in Wuhan, China. Lancet. http://dx.doi.org/10.1016/S0140-6736(20)30183-5. [2020-01-24]. 1

[6] DS. Hui, EI Azhar, TA. Madani, F. Ntoumi, R. Kock, O. Dar, et al. The continuing 2019-nCoV epidemic threat of novel coronaviruses to global health - the latest 2019 novel coronavirus outbreak in Wuhan, China. Int J Infect Dis 2020; 91(2020): 264 - 6. http://dx.doi.org/10.1016/j.ijid.2020.01.009. 1

[7] VJ. Munster, M. Koopmans, N. van Doremalen, D. van Riel, E. de Wit. A novel coronavirus emerging in China - key questions for impact assessment. N Engl J Med. http://dx.doi.org/10.1056/NEJMp2000929. [2020-01-24]. 1

[8] CI. Paules, HD. Marston, AS. Fauci. Coronavirusinfection-more than just the common cold. JAMA. http://dx.doi.org/10.1001/jama. 2020.0757. [2020-01-23]. 1

[9] WJ. Tan, X. Zhao, XJ. Ma, WL. Wang, PH. Niu, WB. Xu, et al. A novel coronavirus genome identified in a cluster of pneumonia cases-Wuhan, China 2019-2020. China CDC Weekly 2020;2(4): 61-2. http://weekly.chinacdc.cn/en/article/ccdcw/2020/4/61. https://doi.org/10.46234/ccdcw2020.017 1 
[10] H. Ugail, Method of trimming PDE surfaces. Computers \& Graphics, (2006), 30, $225-232$. https://doi.org/10.1016/j.cag.2006.01.028

[11] H. W. Vayo, Numerical result onred blood cell geometry, Jpn. J. Physiol., (1982), 32, 891-894. https:/ / doi.org/10.2170/jjphysiol.32.891 1

[12] Wuhan Municipal Health Commission. Report of clustering pneumonia of unknown etiology in Wuhan City. Wuhan, China: Wuhan Municipal Health Commission. http://wjw.wuhan.gov.cn/front/web/ show Detail /2019123108989. [2019-12-31]. (In Chinese). 2

[13] World Health Organization. Novel coronavirus - China. Geneva, Switzerland: World Health Organization. https://www.who.int/csr/don/12-january-2020-novel-corona virus-china/en/. [2020-01-12]. 1

[14] C. Wang, PW. Hornby, FG. Hayden, GF. Gao. A novel coronavirus outbreak of global health concern. Lancet. http://dx.doi.org/10.1016/S0140-6736(20)30185-9. [2020-01-24]. 1

[15] N. Zhu, D. Zhang, W. Wang, XW. Li, B. Yang, JD. Song, et al. A novel coronavirus from patients with pneumonia in China, 2019. N Engl J Med. http://dx. doi.org/10.1056/NEJMoa2001017. [2020-01-24]. 1 1 